\title{
Recreational drug use in heterosexual men in a sexual health clinic in East London: the forgotten majority?
}

Ranjit Samra, Palwasha Khan, Ciara Bansal, Jake Bayley

Barts NHS Trust, London

Introduction: Recreational drug use is widely reported in the MSM population, however its use in the male heterosexual population is less well-described. We undertook a short survey to determine the prevalence of chemsex use in all men.

Methods: Self-directed questionnaires were given to all male attendees at a GUM clinic for three weeks in December 2016. Data on demographics and drug use (including 'chemsex' drugs) was collected.

\section{Results:}

- 268 questionnaires were returned - 70\% were heterosexual and $63 \%$ were of white ethnicity.

- There was much less use of 'chemsex' drugs in heterosexuals versus MSM (20\% versus $9 \%, p=0.03)$. Use of crystal methamphetamine and GHB were much lower in the heterosexual population.

- The highest prevalence of any previous drug use was found in white men vs non-white men (73/133 (55\%) versus 11/65

$(17 \%), p<0.05)$ in both heterosexual and MSM groups.

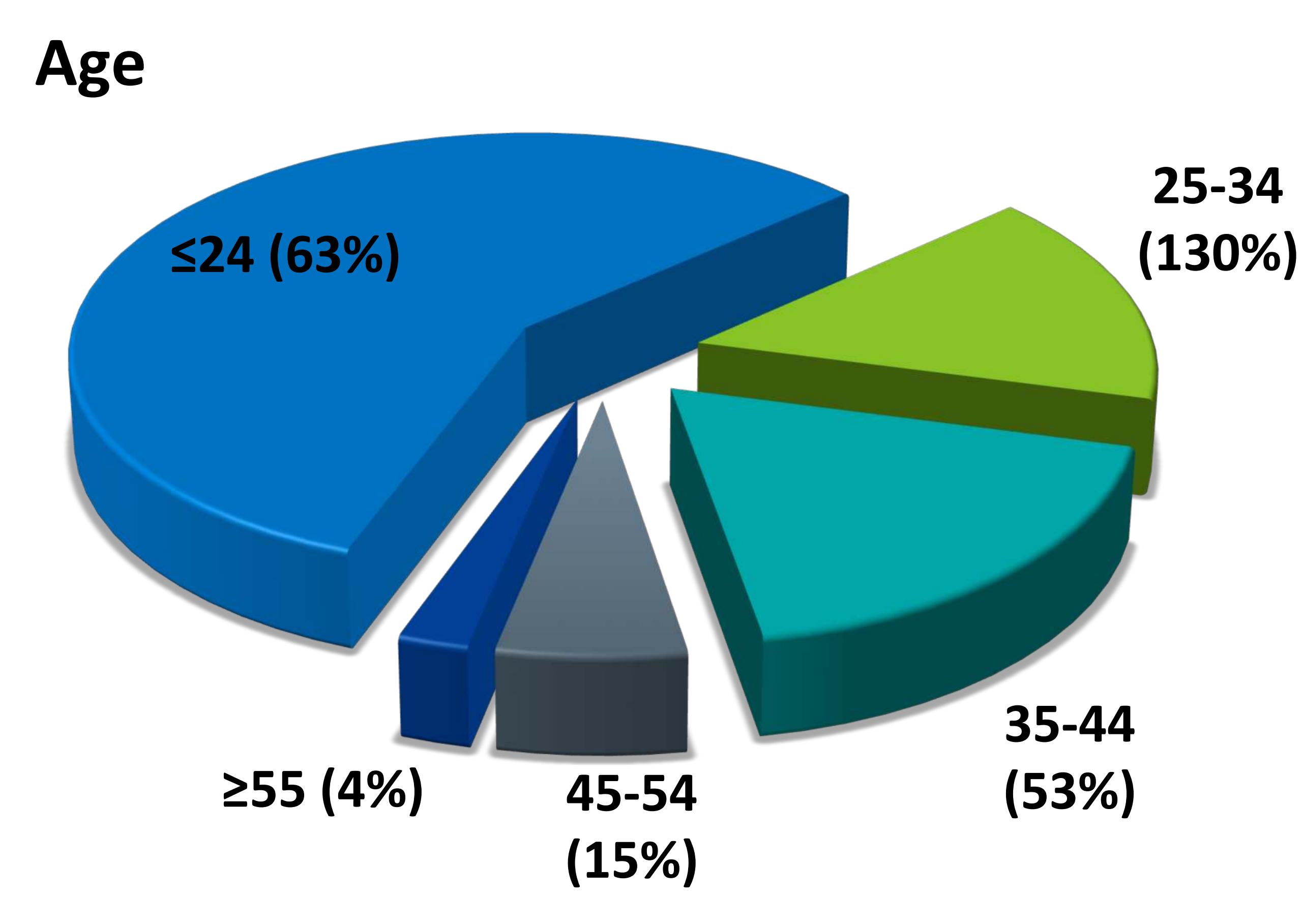

Comparison of Drugs Used in Hetero and MSM Populations
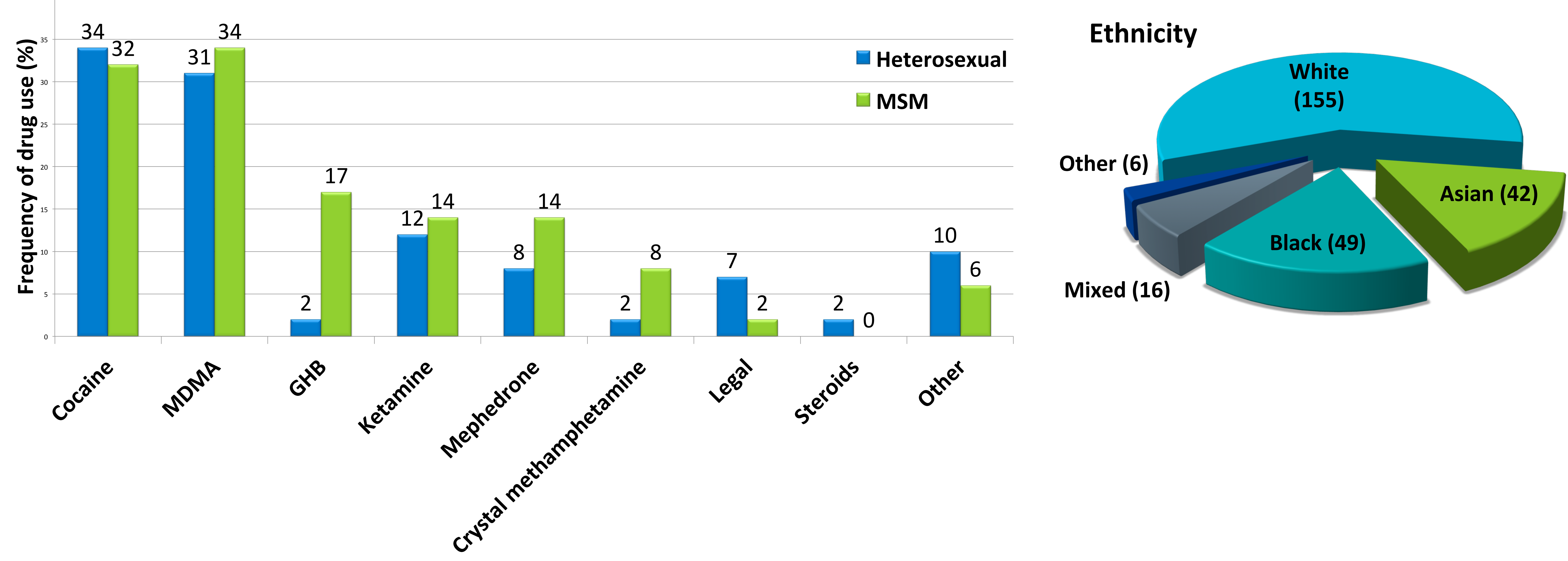

Discussion: There were surprisingly high levels of recreational drug use in heterosexual men, especially those of white ethnicity. 'Chemsex' drugs still seem to be much more common amongst MSM, especially crystal methamphetamine and GHB, but the difference in mephedrone use is much less marked. These data highlight the necessity of asking all patients that attend GUM clinics about their drug use, and not only MSM.

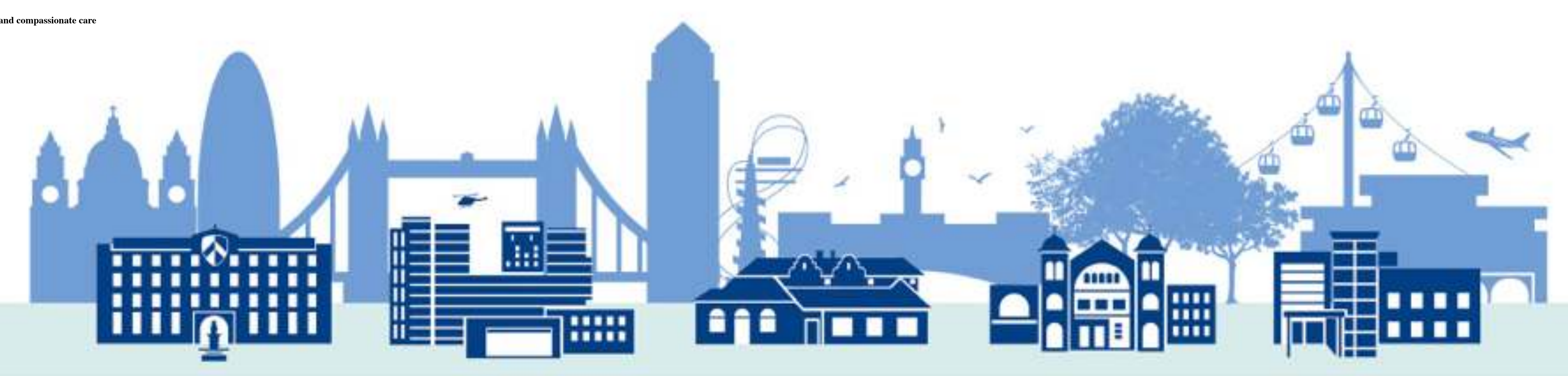

